LBNL-42783

\title{
Laser Acceleration in Vacuum and Gases with Capillary Waveguide
}

\author{
Ming Xie \\ Lawrence Berkeley National Laboratory \\ Berkeley, California 94720, USA \\ Submitted to Physical Review Letters on \\ February 11, 1999
}




\title{
Laser Acceleration in Vacuum and Gases with Capillary Waveguide
}

\author{
Ming Xie \\ Lawrence Berkeley National Laboratory, Berkeley, California 94720
}

(February 11, 1999)

A unified framework is developed to overcome simultaneously three major limitations on acceleration distance and hence on the feasibility of two classes of laser acceleration. The three limitations are due to laser diffraction, acceleration phase slippage, and structure damage by high power laser if solid-state optical waveguide is used. The two classes of laser acceleration are direct-field acceleration and ponderomotivedriven acceleration. This letter and its companion [1] provide solutions that are crucial to all mainstream approaches for laser acceleration, either in vacuum, gases or plasmas.

PACS numbers: 41.75.Jv, 41.75.Lx, 41.75.Ht, 52.40.Fd

\section{INTRODUCTION}

The tremendous growth in accelerator energy of this century has been truly remarkable. However, the workhorse behind this success, microwave powered structure, has more or less reached its known limit in acceleration gradient. To continue the role well into next century as a primary instrument for exploration of fundamental physics in high energy frontier, accelerator has to be powered by electromagnetic (EM) wave of much shorter wavelength. Scaling current technology such as structure dimension down with wavelength is not an attractive pass, at least in the long run. The demand for high gradient acceleration has stimulated widespread interest and research on laser accelerations that operate under fundamentally different concepts and principles.

Yet, despite the impressive progress made in recent years [2], high gradient has been demonstrated only over very short acceleration distances, up to a couple of centimeters. Standing in between proof of principles and real-world applications are three major limitations [3-10]. First of all, the distance of laser-particle interaction is limited by laser diffraction. Secondly, the distance over which a particle can continuously gain energy from a wave is limited by acceleration phase slippage due to the difference between particle speed and phase velocity of the wave. Finally, an acceleration structure, if capable of both guiding laser field and harnessing energy transfer, has to withstand high power laser, and its effectiveness is limited by laser damage threshold. Evidently, the key entry for a breakthrough is at the last point.

In this letter and its companion [1] we present a unified framework to overcome all three major limitations on laser acceleration. Based on interaction mechanism, laser acceleration can be divided into two classes: direct acceleration with the longitudinal field, and acceleration driven by ponderomotive force of the transverse field of a laser. Accordingly, the two classes will be treated separately by the two letters. However, the following discussion on optical waveguide applies to both.

\section{PROPERTIES OF WAVEGUIDE MODES}

The capillary waveguide considered here is made of a hollow core with an index of refraction $\nu_{1}$ and radius $R$, embedded in a medium of dielectric or metal with a complex index of refraction $\nu_{2}$. We are interested in oversized waveguide satisfying $\lambda_{1} / R \ll 1$, where $\lambda_{1}=\lambda / \nu_{1}$ and $\lambda$ is the wavelength in vacuum. As a result, EM wave in the core is dominantly transverse. Capillary waveguide of this type has been used widely for industrial and medical applications to transport lasers of high average power [11]. More recently, it has also been tested at high peak power $[12,13]$. In this section we explore and elaborate the mode properties important for laser acceleration.

The eigenmodes of the waveguide can be solved following the same procedure by Marcatili et al. [14] under the condition $\left|\sqrt{\hat{\nu}^{2}-1}\right| \gg \lambda_{1} / R$, where $\hat{\nu}=\nu_{2} / \nu_{1}$. Expressing the eigenmodes in the following form

$$
\left\{\begin{array}{l}
\mathcal{E}(r, \phi, z, t) \\
\mathcal{H}(r, \phi, z, t)
\end{array}\right\}=\left\{\begin{array}{c}
\mathbf{E}_{l m}(r, \phi) \\
\mathbf{H}_{l m}(r, \phi)
\end{array}\right\} e^{i\left(\beta_{l m} z-\omega t\right)-\alpha_{l m} z},
$$

the eigenvalues are given by

$$
\beta_{l m}=k_{1}\left(1-1 / 2 \gamma_{g}^{2}\right), \quad \alpha_{l m}=\operatorname{Re}(\Lambda) / \gamma_{g}^{2} R,
$$

where $k_{1}=\nu_{1} k, k=2 \pi / \lambda, \gamma_{g}=2 \pi R / U_{l m} \lambda_{1} \gg 1$, and $U_{l m}$ is the $m$ th root of the equation $J_{l-1}\left(U_{l m}\right)=0$. There are three types of modes, corresponding to

$$
\Lambda=\left\{\begin{array}{lll}
\frac{1}{\sqrt{\hat{\nu}^{2}-1}} & : & T E_{0 m}(l=0) \\
\frac{\hat{\nu}^{2}}{\sqrt{\hat{\nu}^{2}-1}} & : & T M_{0 m}(l=0) \\
\frac{\hat{\nu}^{2}+1}{2 \sqrt{\hat{\nu}^{2}-1}} & : & E H_{l m}(l \neq 0) .
\end{array}\right.
$$

For laser acceleration, we are interested primarily in two low-order modes: $T M_{01}$ mode for direct-field acceleration in vacuum and gases with its on-axis $E_{z}$ component, and $E H_{11}$ mode for ponderomotive-driven acceleration in plasmas. Accordingly, we consider three cases: $\delta \nu_{1}=0$ when the core is in vacuum, $\delta \nu_{1}>0$ and $\delta \nu_{1}<0$ when the core is filled with uniform gases and plasmas, respectively, where $\delta \nu_{1}=\nu_{1}-1$ and $\left|\delta \nu_{1}\right| \ll 1$. 
The electric fields within the core $r \leq R$ are given by

$$
\begin{aligned}
& T M_{01}:\left\{\begin{array}{l}
E_{z}=E_{a} J_{0}\left(k_{r 1} r\right) \\
E_{r}=-i\left(\Gamma / k_{r 1}\right) E_{a} J_{1}\left(k_{r 1} r\right),
\end{array}\right. \\
& E H_{11}:\left\{\begin{array}{l}
E_{y}=E_{0} J_{0}\left(k_{r 1} r\right) \\
E_{z}=-i\left(k_{r 1} / \Gamma\right) E_{0} J_{1}\left(k_{r 1} r\right) \sin \phi,
\end{array}\right.
\end{aligned}
$$

where $E_{a}$ is the peak acceleration field for $T M_{01}$ mode, $E_{0}$ is the peak transverse field for $E H_{11}$ mode, $\Gamma=$ $\beta_{l m}+i \alpha_{l m}$ and $k_{r 1}=\left(U_{l m}-i \Lambda / \gamma_{g}\right) / R$. To leading order $\Gamma / k_{r 1}=\gamma_{g}$, since by definition $k_{1}^{2}=\Gamma^{2}+k_{r 1}^{2}$. Given electric field, magnetic field of a mode can be determined by $\mathbf{H}_{t}=\widehat{\mathbf{z}} \times\left(\Gamma \mathbf{E}_{t}+i \nabla_{t} E_{z}\right) / k Z_{0}, H_{z}=(i / \Gamma) \nabla_{t} \cdot \mathbf{H}_{t}$, where subscript $t$ denotes transverse component of a vector or operator, $\hat{\mathbf{z}}$ is a unit vector in z-direction, and $Z_{0}$ is the vacuum impedance. As seen from Eqs. $(4,5)$, the transverse fields dominate over the longitudinal ones by a large factor $\gamma_{g}$. For $T M_{01}$ mode, $E_{r}$ is peaked at $r / R=0.481$ with a maximum value $E_{r}^{\max }=0.582 \gamma_{g} E_{a}$. For $E H_{11}$ mode, $E_{y}$ is peaked on the axis. To evaluate surface field $E_{s}$ at $r=R$, we expand Bessel functions in the transverse fields of Eqs. $(4,5)$ using the expression for $k_{r 1}$ and keeping the larger one of the two components

$$
\begin{aligned}
& E_{s} / E_{a}=\max \{1,|\Lambda|\}\left|J_{0}\left(U_{01}\right)\right| \quad: T M_{01}, \\
& E_{s} / E_{0}=\max \{1,|\Lambda|\}\left|J_{1}\left(U_{11}\right)\right| / \gamma_{g}: E H_{11} \text {. }
\end{aligned}
$$

Here we come upon one of the most important advantages of the capillary waveguide: for $T M_{01}$ mode, surface field can be smaller than peak acceleration field, superior to other schemes [4-10] including even microwave structure; and for both modes, surface fields are much smaller than peak transverse fields. According to experimental data on dielectrics at $\lambda=1 \mu \mathrm{m}$ [15], damage threshold in terms of surface field is around $5 \sim 10 \mathrm{GV} / \mathrm{m}$ for pulse length of 1 ps or shorter, thus making acceleration in $\mathrm{GV} / \mathrm{m}$ scale possible. Within the cladding $r \geq R$, all field components have the radial dependence $e^{i k_{r 2} r} / \sqrt{r}$, where to leading order $k_{r 2}=k_{1} \sqrt{\hat{\nu}^{2}-1}$. A non-vanishing imaginary part of $\hat{\nu}$ due to dielectric loss would give rise to exponential decay in radial direction. Hence power in each mode is distributed dominantly within the core and it can be expressed as $P(z)=P_{0} e^{-z / L_{a t t n}}$, where $L_{a t t n}=1 / 2 \alpha_{l m}$ is power attenuation length and to leading order

$$
P_{0}=\left\{\begin{array}{lll}
\pi R^{2} \gamma_{g}^{2} E_{a}^{2} J_{0}\left(U_{01}\right)^{2} / 2 Z_{0} & : & T M_{01} \\
\pi R^{2} E_{0}^{2} J_{1}\left(U_{11}\right)^{2} / 2 Z_{0} & : & E H_{11}
\end{array} .\right.
$$

It is noted that $E H_{11}$ mode is linearly polarized, whereas $T M_{01}$ mode is radially polarized. A linearly polarized mode with non-vanishing on-axis $E_{z}$ can be constructed by a proper mixing of $T M_{01}$ with $E H_{21}$ mode [16]. Electric field for the mixed mode is given by

$$
\begin{aligned}
& E_{z}=E_{a}\left[J_{0}\left(k_{r 1} r\right)+J_{2}\left(k_{r 1} r\right) \cos 2 \phi\right] \\
& E_{y}=-2 i\left(\Gamma / k_{r 1}\right) E_{a} J_{1}\left(k_{r 1} r\right) \sin \phi .
\end{aligned}
$$

To preserve the same acceleration field $E_{a}$ as $T M_{01}$ mode, the mixed mode requires a factor of two more power.
For the three modes we have $U_{11}=2.405, U_{01}=U_{21}$ $=3.832, J_{0}\left(U_{01}\right)=-0.403$ and $J_{1}\left(U_{11}\right)=0.519$. Coupling between the waveguide modes and Gauss-Laguerre modes, also known as $T E M_{p l}$ modes [11], can be very efficient. When focused at waveguide entrance, power coupling from a radially polarized $T E M_{01}$ mode to the $T M_{01}$ mode reaches a maximum of $97 \%$ at $w_{0} / R=0.56$. This is true also for coupling from a linearly polarized $T E M_{01}$ mode to the mixed $T M_{01}+E H_{21}$ mode. Coupling from a $T E M_{00}$ mode to the $E H_{11}$ mode is $98 \%$ at $w_{0} / R=0.64$, where $w_{0}$ is the Gaussian mode waist.

The on-axis intensity for a $T E M_{00}$ mode falls as $I(z) / I(0)=1 /\left[1+\left(z / Z_{R}\right)^{2}\right]$ away from the waist due to diffraction, and the on-axis longitudinal field for a $T E M_{01}$ mode also falls as $E_{z}(z) / E_{z}(0)=1 /\left[1+\left(z / Z_{R}\right)^{2}\right]$, where $Z_{R}=\pi w_{0}^{2} / \lambda_{1}$ is the Rayleigh length. Assuming a $T E M_{00}\left(T E M_{01}\right)$ mode is coupled to a $E H_{11}\left(T M_{01}\right)$ mode at the optimal condition, the effectiveness of guiding can be measured by taking the ratio of the relevant e-folding lengths of the waveguide mode to the free-space mode, yielding $L_{E H 11} / L_{T E M 00}=L_{T M 01} / L_{T E M 01}=$ $2 R / \operatorname{Re}(\Lambda) \lambda_{1}$. Despite the fact that the waveguide modes are leaky, optical guiding can be made quite effective to overcome diffraction for low order modes with sufficiently large $R / \lambda_{1}$. In addition, for waveguide material with anomalous dispersion at certain wavelength, it is possible to have $\operatorname{Re}(\Lambda) \ll 1$. For example, we have $\operatorname{Re}(\Lambda)=0.1$ for sapphire at $\lambda=10.6 \mu \mathrm{m}$ with $\nu_{2}=0.67+i 0.04$ [17].

\section{ACCELERATION IN VACUUM}

According to Eq.(2), phase velocity of the $T M_{01}$ mode is $v_{p}=\omega / \beta_{01}=c /\left(1-1 / 2 \gamma_{g}^{2}\right)$, larger than the speed of light. We define an acceleration phase slippage length by

$$
L_{a}=\frac{\lambda}{1 / \gamma_{g}^{2}+1 / \gamma^{2}},
$$

over which a relativistic electron with energy $W_{0}=\gamma m c^{2}$, while being accelerated, slips $\pi$ phase with respect to the fast acceleration wave. Energy gain of the electron on the axis is $\Delta W_{a}=e E_{a} \int_{0}^{L_{a}} \sin \left(\pi z / L_{a}\right) d z=e E_{a} L_{a} T_{a}$, where $T_{a}=2 / \pi$ is a transit factor. Here we have neglected the small waveguide attenuation over the distance $L_{a}$. In parallel, we may also define a deceleration slippage length over which the electron slips another $\pi$ phase while losing energy amounted to $\Delta W_{d}=e E_{a} L_{d} T_{d}$. Average acceleration during a period of $2 \pi$ phase slippage is then

$$
G=\frac{\Delta W_{a}-\Delta W_{d}}{L_{a}+L_{d}}=\frac{e E_{a} T_{a}\left[1-\left(L_{d} / L_{a}\right)\left(T_{d} / T_{a}\right)\right]}{1+L_{d} / L_{a}} .
$$

To have net acceleration, the ratio $L_{d} / L_{a}$ should be made small. Two methods are proposed here for this purpose. The idea is to enhance phase slippage during the half period of deceleration, thus taking a shorter distance $L_{d}$. 
The first method works on reducing the longitudinal velocity of an electron by introducing a static transverse magnetic field during deceleration. Without loss of generality, we assume the magnetic field is sinusoidal as in a wiggler $B_{y}=B_{0} \cos \left(2 \pi z / \lambda_{w}\right)$ with a period $\lambda_{w}$. Hence by definition $L_{d}$ is determined through the condition

$$
\left[\frac{1}{\gamma_{g}^{2}}+\frac{1}{\gamma^{2}}+\frac{a_{w}^{2}}{\gamma^{2}}\right] \frac{\pi L_{d}}{\lambda}-\frac{a_{w}^{2} \lambda_{w}}{4 \gamma^{2} \lambda} \sin \left[\frac{4 \pi L_{d}}{\lambda_{w}}\right]=\pi,
$$

where $a_{w}=e B_{0} \lambda_{w} / 2 \pi \sqrt{2} m c$. If we set $\lambda_{w}=L_{d}$, then

$$
L_{d}=\frac{\lambda}{1 / \gamma_{g}^{2}+1 / \gamma^{2}+a_{w}^{2} / \gamma^{2}}
$$

here now $a_{w}=\sqrt[3]{Q_{1}+\sqrt{Q_{1}^{2}+Q_{2}^{3}}}+\sqrt[3]{Q_{1}-\sqrt{Q_{1}^{2}+Q_{2}^{3}}}$, with $Q_{1}=e B_{0} \lambda \gamma^{2} / 4 \pi \sqrt{2} m c$ and $Q_{2}=\left[1+\left(\gamma / \gamma_{g}\right)^{2}\right] / 3$. Due to longitudinal oscillation in electron orbit, $T_{d} / T_{a}=$ $\frac{1}{2} \int_{0}^{\pi} \sin \left[\theta-\sin (4 \theta)\left(1-L_{d} / L_{a}\right) / 4\right] d \theta$, which varies within the range $\{0.92 \leftrightarrow 1\}$ for $L_{d} / L_{a}$ in the full range $\{0 \leftrightarrow 1\}$. The radiative energy loss per wiggler period is $\Delta W_{r}=$ $\left(8 \pi^{2} m c^{2} / 3\right)\left(r_{e} / \lambda_{w}\right) a_{w}^{2} \gamma^{2}$, where $r_{e}$ is the classical radius of electron. The maximum possible energy that can be accelerated with this method is restricted by the condition $\Delta W_{a}>\Delta W_{d}+\Delta W_{r}$.

Table 1. Example in Vacuum with Magnetic Field.

\begin{tabular}{|c|c|c|c|c|c|}
\hline$\lambda[\mu \mathrm{m}]$ & 1 & $P_{0}[\mathrm{TW}]$ & 50 & $E_{a}[\mathrm{GV} / \mathrm{m}]$ & 1.8 \\
\hline$R / \lambda$ & 300 & $B_{0}[\mathrm{~T}]$ & 3 & $E_{s}[\mathrm{GV} / \mathrm{m}]$ & 1.5 \\
\hline$\nu_{2}$ & 1.5 & $a_{w}$ & 22 & $I_{s}\left[\mathrm{TW} / \mathrm{cm}^{2}\right]$ & 0.3 \\
\hline$\gamma_{g}$ & 492 & $\Delta X_{\max } / R$ & 0.37 & $G[\mathrm{GeV} / \mathrm{m}]$ & 0.45 \\
\hline$W_{0}[\mathrm{GeV}]$ & 5 & $L_{a}[\mathrm{~cm}]$ & 24 & $\Delta W_{a}[\mathrm{MeV}]$ & 283 \\
\hline$\beta_{t}[\mathrm{~cm}]$ & 46 & $L_{d}[\mathrm{~cm}]$ & 11 & $\Delta W_{d}[\mathrm{MeV}]$ & 124 \\
\hline$L_{\text {attn }}[\mathrm{m}]$ & 18 & $T_{d} / T_{a}$ & 0.96 & $\Delta W_{r}[\mathrm{keV}]$ & 16 \\
\hline
\end{tabular}

Transverse EM force on a relativistic electron does not vanish to order of $1 / \gamma^{2}$ in a waveguide mode or when the index of refraction differs from unity. For the $T M_{01}$ mode with magnetic field $H_{\phi}=\left(1+1 / 2 \gamma_{g}^{2}+\delta \nu_{1}\right) E_{r} / Z_{0}$, transverse force is $F_{r}=-e\left(1 / 2 \gamma^{2}-1 / 2 \gamma_{g}^{2}-\delta \nu_{1}\right) E_{r}$, which may lead to either focusing or defocusing depending on acceleration phase $\phi_{a}$. Correspondingly, the beta function for an electron near the axis in vacuum is

$$
\beta_{t}=\gamma_{g} \lambda \sqrt{\left(\gamma m c^{2} / \pi e \lambda E_{a} \sin \phi_{a}\right) /\left[1-\left(\gamma_{g} / \gamma\right)^{2}\right]}
$$

The condition for an electron to remain near the axis may not be satisfied in the deceleration section, where the maximum orbital offset in x-direction is $\Delta X_{\max }=$ $\sqrt{2} a_{w} \lambda_{w} / \pi \gamma$. To avoid strong nonlinear transverse EM force while an electron is deflected far off-axis by the wiggler field, the mixed mode $T M_{01}+E H_{21}$ may be used, for it has zero transverse field along x-axis. An example is given in Table 1 for acceleration in vacuum with $T M_{01}$ mode, where in calculating $\beta_{t}$ we set $\sin \phi_{a}=1$, and $I_{s}$ is laser intensity on waveguide inner surface.

Instead of tempering electron orbit, the second method works on enhancing phase slippage by increasing phase velocity of the wave during deceleration. This can be done by introducing a plasma layer of thickness

$$
L_{d}=\frac{\lambda}{1 / \gamma_{g}^{2}+1 / \gamma^{2}+1 / \gamma_{p}^{2}},
$$

where $\gamma_{p}=\omega / \omega_{p} \gg 1, \omega_{p}=c \sqrt{4 \pi r_{e} n_{0}}$ is the electron plasma frequency and $n_{0}$ is the plasma density. Here we have used for the plasma an index of refraction $\nu_{1}=$ $1-\omega_{p}^{2} / 2 \omega^{2}$. In this case, $T_{d}=T_{a}$. The dominant energy loss for an ultrarelativistic electron traversing a plasma is due to bremsstrahlung [18]. The rate of energy loss is given by $d W / d z=-W / L_{R}$, where $L_{R}$ is the radiation length defined by $1 / L_{R}=4 \alpha r_{e}^{2} n_{i} Z(Z+1) \ln \left(233 / Z^{1 / 3}\right)$, $n_{i}$ is density of ions with atomic number $Z$, and $\alpha$ is the fine structure constant. For Hydrogen plasma with density $n_{i}=n_{0}=10^{17} / \mathrm{cm}^{3}, L_{R}$ is as long as $4 \times 10^{6} \mathrm{~m}$. Reflectance of laser power off a sharp interface between vacuum and an underdense plasma at normal incidence is also negligible, according to the Fresnel formula $R_{p}=$ $\left(1-\nu_{1}\right)^{2} /\left(1+\nu_{1}\right)^{2}=1 / 16 \gamma_{p}^{4}$. Two examples are given in Table 2 and Table 3 for highly relativistic electron satisfying $\left(\gamma / \gamma_{g}\right)^{2} \gg 1$ and $\left(\gamma / \gamma_{p}\right)^{2} \gg 1$.

Table 2. Example in Vacuum with Plasma Layer (I).

\begin{tabular}{|c|c|c|c|c|c|}
\hline$\lambda[\mu \mathrm{m}]$ & 1 & $P_{0}[\mathrm{TW}]$ & 10 & $E_{a}[\mathrm{GV} / \mathrm{m}]$ & 1.9 \\
\hline$R / \lambda$ & 200 & $n_{0}\left[10^{17} / \mathrm{cm}^{3}\right]$ & 1.1 & $E_{s}[\mathrm{GV} / \mathrm{m}]$ & 1.5 \\
\hline$\nu_{2}$ & 1.5 & $L_{a t t n}[\mathrm{~m}]$ & 5.3 & $G[\mathrm{GeV} / \mathrm{m}]$ & 1 \\
\hline$\gamma_{g}$ & 328 & $L_{a}[\mathrm{~cm}]$ & 10.8 & $\Delta W_{a}[\mathrm{MeV}]$ & 127 \\
\hline$\gamma_{p}$ & 100 & $L_{d}[\mathrm{~cm}]$ & 0.91 & $\Delta W_{d}[\mathrm{MeV}]$ & 11 \\
\hline
\end{tabular}

Table 3. Example in Vacuum with Plasma Layer (II).

\begin{tabular}{|c|c|c|c|c|c|}
\hline$\lambda[\mu \mathrm{m}]$ & 10.6 & $P_{0}[\mathrm{TW}]$ & 5 & $E_{a}[\mathrm{GV} / \mathrm{m}]$ & 2.0 \\
\hline$R / \lambda$ & 50 & $n_{0}\left[10^{17} / \mathrm{cm}^{3}\right]$ & 0.11 & $E_{s}[\mathrm{GV} / \mathrm{m}]$ & 0.8 \\
\hline $\operatorname{Re}\left(\nu_{2}\right)$ & 0.67 & $L_{a t t n}[\mathrm{~m}]$ & 18 & $G[\mathrm{GeV} / \mathrm{m}]$ & 1 \\
\hline $\operatorname{Im}\left(\nu_{2}\right)$ & 0.04 & $L_{a}[\mathrm{~cm}]$ & 7.1 & $\Delta W_{a}[\mathrm{MeV}]$ & 90 \\
\hline$\gamma_{p}$ & 30 & $L_{d}[\mathrm{~cm}]$ & 0.84 & $\Delta W_{d}[\mathrm{MeV}]$ & 11 \\
\hline
\end{tabular}

The assumption of a sharp vacuum-plasma interface is not necessary. More rigorous treatment can be obtained with WKB method [19]. For underdense plasma, the only modification required is to replace the factor $\exp \left(i \beta_{01} z\right)$ by $\exp \left[i \int \beta_{01}(z) d z\right]$. Assuming a density profile $n_{e}=$ $n_{0} f_{p}(z)$, where $f_{p}(z)=1 /\left[1+e^{-\left(z+L_{d} / 2\right) / \delta}\right]-1 /[1+$ $\left.e^{-\left(z-L_{d} / 2\right) / \delta}\right]$, the phase advance for the mode can be 
calculated by making use of the integral $\int_{-\infty}^{\infty} f_{p}(z) d z=$ $2 \delta \ln \left[\left(1+e^{L_{d} / 2 \delta}\right) /\left(1+e^{-L_{d} / 2 \delta}\right)\right]$. It is seen here that Eq.(14) is accurate enough as long as $L_{d} / 2 \delta \gg 1$. In addition, the validity of WKB method requires $\left|d \nu_{1} / d z\right| \ll$ $2 \pi \nu_{1}^{2} / \lambda[19]$, which gives $\delta \gg \lambda / 16 \pi \gamma_{p}^{2}$ for $d f_{p} /\left.d z\right|_{\max }=$ $1 / 4 \delta$, also easily satisfied. To implement plasma layers in sections of a waveguide, side access is preferred. This can be done by inserting sections of open iris-loaded waveguide [20] wherever necessary. The mode profile in such a waveguide is nearly identical to that of a capillary waveguide, allowing significant minimization of mode coupling loss due to waveguide interruption.

\section{ACCELERATION IN GASES}

The phase velocity of the $T M_{01}$ mode in a core of uniform neutral gases is $v_{p}=\omega / \beta_{01}=c /\left(1-1 / 2 \gamma_{g}^{2}+\right.$ $\left.\delta \nu_{1}\right)$, corresponding to a phase slippage length $L_{a}=$ $\lambda /\left|1 / \gamma_{g}^{2}+1 / \gamma^{2}-2 \delta \nu_{1}\right|$. The phase matching condition for synchronous acceleration is obtained by making the denominator zero, thus $\delta \nu_{1}=1 / 2 \gamma_{g}^{2}+1 / 2 \gamma^{2}$. This condition suggests an alternative way to maintain phase matching as $\gamma$ increases during acceleration: instead of varying $\delta \nu_{1}$ by adjusting gas pressure along the waveguide, $\gamma_{g}$ may be changed by tapering waveguide radius. For highly relativistic electron satisfying $\left(\gamma / \gamma_{g}\right)^{2} \gg 1$, a steady-state phase matching condition, $\delta \nu_{1}=1 / 2 \gamma_{g}^{2}$, is approached. An example is given in Table 4 in this limit. The beta function is smaller by a factor of $\sqrt{2}$ than that given for vacuum by Eq.(13). The maximum acceleration gradient achievable is limited by various processes occurring in gases in the field of high power laser, such as gas breakdown. Here we assume the limit is set on the maximum field for the $T M_{01}$ mode by $E_{r}^{\max } \leq 10 \mathrm{GV} / \mathrm{m}$.

Table 4. Example in Gases.

\begin{tabular}{|c|c|c|c|c|c|}
\hline$\lambda[\mu \mathrm{m}]$ & 10.6 & $\operatorname{Re}\left(\nu_{2}\right)$ & 0.67 & $E_{a}[\mathrm{GV} / \mathrm{m}]$ & 0.21 \\
\hline$R / \lambda$ & 50 & $\operatorname{Im}\left(\nu_{2}\right)$ & 0.04 & $E_{s}[\mathrm{GV} / \mathrm{m}]$ & 0.085 \\
\hline$P_{0}[\mathrm{GW}]$ & 56 & $\delta \nu_{1}\left[10^{-5}\right]$ & 7.4 & $L_{\text {attn }}[\mathrm{m}]$ & 18 \\
\hline
\end{tabular}

Among other schemes of synchronous acceleration, one uses a line focus from an axicon in gases $[7,8]$. In comparison, our approach requires less laser power and provides much longer acceleration distance for the same $\gamma, \delta \nu_{1}$ and $E_{a}$. Another scheme uses a dielectrically lined perfect conductor tube as a lossless waveguide for TM modes $[9,10]$. This scheme, first of all, belongs to the class of acceleration by evanescent field near a surface of dielectric [3] and its scaling, $E_{a} / E_{s}=\lambda / \pi R$, is unfavorable for laser acceleration. Furthermore, the assumption of perfect conductor is no longer valid for metals at wavelength as short as $\lambda=10.6 \mu \mathrm{m}$, where circular metallic waveguide is known to be very lossy for TM modes [18].

\section{CONCLUSIONS}

I have introduced the concepts and techniques that are crucial for advancing the current development of laser acceleration into a new, more realistic stage. Of what being accomplished here, the most notable is the dramatic increase in single-stage acceleration distance comparing with other schemes [4-10] for direct-field acceleration. This breakthrough is achieved in two critical steps. First, the diffraction limit is overcome with the capillary waveguide. Second, the phase slippage limit is overcome in vacuum with two new mechanisms for energy transfer. The two steps in combination succeed in harnessing high power laser to achieve high gradient with durable structure. In comparison, other schemes are severely limited for having components being blasted by full laser power in close range, head-on [4-6]. Furthermore, the approach taken here has the following advantages: oversized structure dimension is favorable for more accelerated particles; electromagnetically open waveguide suppresses higher order modes in favor of better beam quality; efficient coupling between waveguide and free-space modes simplifies mode handling such as injection, transport and recycling, leading to better overall system efficiency; last but not least, all aforementioned features are achieved without sacrificing a virtue of practical importance: the simplicity. Beam dynamics and collective effects will be addressed in a forthcoming series of papers. This work was supported by the U.S. Department of Energy under contract No.DE-AC03-76SF00098.

[1] M. Xie, Laser Acceleration in Plasmas with Capillary Waveguide, LBNL-42784, submitted to Phys. Rev. Lett. on February 11, 1999.

[2] AIP Conf. Proc., 335 (1995), 398 (1997), 472 (1999).

[3] R. Palmer, AIP Conf. Proc. 335, 90 (1995).

[4] E. Esarey et al., Phys. Rev. E, 52, 5443 (1995).

[5] Y. Huang et al., Appl. Phys. Lett. 68, 753 (1996).

[6] L. Steinhauer et al., J. Appl. Phys., 83, 5636 (1998).

[7] J. Fontana et al., J. Appl. Phys., 54, 4285 (1983).

[8] W. Kimura et al., Phys. Rev. Lett. 74, 546 (1995).

[9] W. Gai et al., AIP Conf. Proc. 398, 564 (1997).

[10] P. Sprangle et al., Phys. Rev. E, 55, 5964 (1997).

[11] N. Hodgson and H. Weber, Optical Resonators, Chapter 5 and 18 (Springer-Verlag, 1997).

[12] S. Jackel et al., Optics Letters, 20, 1086 (1995).

[13] C. Durfee et al., Optics Letters, 22, 1565 (1997).

[14] E. Marcatili et al., B.S.T.J., 43, 1783 (1964).

[15] B. Stuart et al., Phys. Rev. Lett, 74, 2248 (1995).

[16] J. Henningsen et al., Appl. Phys., B51, 272 (1990).

[17] C. Gregory et al., Applied Optics, 32, 5302 (1993).

[18] J. Jackson, Classical Electrodynamics, 2nd edition, Chapter 8 and 15 (John Wiley \& Sons, 1975).

[19] V. Ginzburg, The Propagation of Electromagnetic Waves in Plasmas, Chapter IV (Pergamon Press, 1964).

[20] M. Xie, Proc. of 1997 Part. Accel. Conf., 660 (1997). 